\title{
Fast simulation of ultrasound images
}

\author{
Jensen, Jørgen Arendt; Nikolov, Svetoslav
}

\section{Published in:}

Proceedings on Ultrasonics Symposium

Link to article, DOI:

10.1109/ULTSYM.2000.921654

Publication date:

2000

Document Version

Publisher's PDF, also known as Version of record

Link back to DTU Orbit

Citation (APA):

Jensen, J. A., \& Nikolov, S. (2000). Fast simulation of ultrasound images. In Proceedings on Ultrasonics Symposium (Vol. 2, pp. 1721-1724). IEEE. https://doi.org/10.1109/ULTSYM.2000.921654

\section{General rights}

Copyright and moral rights for the publications made accessible in the public portal are retained by the authors and/or other copyright owners and it is a condition of accessing publications that users recognise and abide by the legal requirements associated with these rights.

- Users may download and print one copy of any publication from the public portal for the purpose of private study or research.

- You may not further distribute the material or use it for any profit-making activity or commercial gain

- You may freely distribute the URL identifying the publication in the public portal

If you believe that this document breaches copyright please contact us providing details, and we will remove access to the work immediately and investigate your claim. 


\title{
Fast Simulation of Ultrasound Images
}

\author{
Jørgen Arendt Jensen and Svetoslav Ivanov Nikolov \\ Center for Fast Ultrasound Imaging, Department of Information Technology, Build. 344, \\ Technical University of Denmark, DK-2800 Lyngby, Denmark
}

\begin{abstract}
Realistic B-mode and flow images can be simulated with scattering maps based on optical, CT, or MR images or parametric flow models. The image simulation often includes using 200,000 to 1 million point scatterers. One image line typically takes 1800 seconds to compute on a state-of-the-art PC, and a whole image can take a full day. Simulating $3 \mathrm{D}$ images and 3D flow takes even more time. A 3D image of 64 by 64 lines can take 21 days, which is not practical for iterative work. This paper presents a new fast simulation method based on the Field II program. In imaging the same spatial impulse response is calculated for each of the image lines, and making 100 lines, thus, gives 100 calculations of the same impulse response delayed differently for the different lines. Doing the focusing after this point in the simulation can make the calculation faster. This corresponds to full synthetic aperture imaging. The received response from each element is calculated, when emitting with each of the elements in the aperture, and then the responses are subsequently focused. This is the approach taken in this paper using a modified version of the Field II program. A 64 element array, thus, gives 4096 responses. For a $7 \mathrm{MHz} 64$ element linear array the simulation time for one image line is 471 seconds for 200,000 scatterers on a $800 \mathrm{MHz}$ AMD Athlon PC, corresponding to 17 hours for one image with 128 lines. Using the new approach, the computation time is 10,963 seconds, and the beamforming time is 9 seconds, which makes the approach 5.5 times faster. For $3 \mathrm{D}$ images with 64 by 64 lines, the total conventional simulation time for one volume is 517 hours, whereas the new approach makes the simulation in 6,810 seconds. The time for beamforming is 288 seconds, and the new approach is, thus, 262 times faster. The simulation can also be split among a number of PCs for speeding up the simulation. A full 3D one second volume simulation then takes 7,500 seconds on a 32 CPU $600 \mathrm{MHz}$ Pentium III PC cluster.
\end{abstract}

\section{Introduction}

The simulation of ultrasound imaging using linear acoustics has been extensively used for studying focusing, image for-

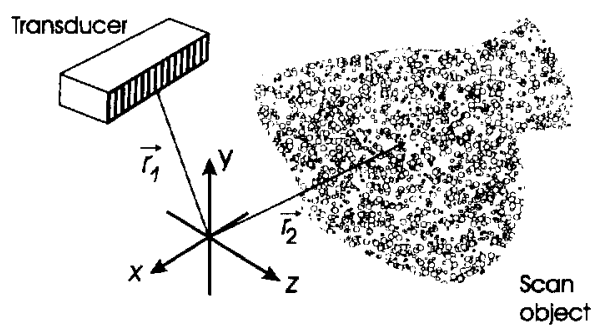

Figure 1: Set-up for simulation of ultrasound imaging.

mation, and flow estimation, and it has become a standard tool in ultrasound research. Simulation, however, still takes a considerable amount of time, when realistic imaging, flow, or 3D imaging are studied. New techniques for reducing the simulation time are, thus, desirable.

The main part of an ultrasound image consists of a speckle pattern, which emanates from the signal generated by tissue cells, connective tissue, and in general all small perturbations in speed of sound, density, and attenuation. The generation of this can be modeled as the signal from a large collection of randomly placed point scatterers with a Gaussian amplitude. Larger structures as vessel or organ boundaries can be modeled as a deterministicly placed set of point scatterers with a deterministic amplitude. The relative amplitude between the different scatterers is then determined by a scatterer map of the structures to be scanned. Such maps can be based on either optical, CT or MR images, or on parametric models of the organs. Currently the most realistic images are based on optical images of the anatomy [1]. Blood flow can also be modeled by this method. The red blood cells, mainly responsible for the scattering, can be modeled as point scatterers and the flow of the blood can be simulated using either a parametric flow mode] [2] or through finite element modeling [3]. The received signal is then calculated, and the scatterers are propagated between flow emissions. The simulation of all linear ultrasound systems can, thus, be done by finding the summed signal from a collection of point scatterers as shown in Fig. 1. The random selection of point scatterers should consist of at least 10 scatterers per resolution cell to generate fully developed speckle, and for a normal ultrasound image this results 
in 200,000 to 1 million scatterers. The simulation of the responses from these scatterers must then be done for each line in the resulting image, and the simulation for the whole collection is typically done 100 times with different delay focusing and apodization. This makes the simulation take several days even on a fast workstation.

A second possibility is to do fully synthetic aperture imaging in which the received response by all elements are found, when transmitting with each of the elements in the array. The response of each element is then only calculated once, and the simulation time can be significantly reduced. This is the approach suggested in this paper. A second advantage of such an approach is that the image is focused after the field simulation. The same data can, thus, be used for testing a number of focusing strategies without redoing the simulation. This makes is easier to find optimized focusing strategies.

\section{Theory}

The field simulation must find the received signal from a collection of point scatterers. Using linear acoustics the received voltage signal is [4]:

$$
v_{r}(t)=v_{p e}(t) \underset{t}{\star} f_{m}\left(\vec{r}_{1}\right) \underset{r}{\star} h_{p e}\left(\vec{r}_{1}, t\right),
$$

where $\stackrel{\star}{r}$ denotes spatial convolution, $\stackrel{\star}{t}$ temporal convolution, and $\vec{r}_{1}$ the position of the point scatterer. $v_{p e}(t)$ is the pulse-echo wavelet, which includes both the transducer excitation and the electro-mechanical impulse response during emission and reception of the pulse. $f_{m}$ accounts for the inhomogeneities in the tissue due to density and speed of sound perturbations that generates the scattering, and $h_{p e}$ is the pulse-echo spatial impulse response that relates the transducer geometry to the spatial extent of the scattered field. Explicitly written out the latter term is:

$$
h_{p e}\left(\vec{r}_{1}, t\right)=h_{t}\left(\vec{r}_{1}, t\right) \underset{t}{\star} h_{r}\left(\vec{r}_{1}, t\right)
$$

where $h_{t}\left(\vec{r}_{1}, t\right)$ is the spatial impulse response for the transmitting aperture and $h_{r}\left(\vec{r}_{1}, t\right)$ is the spatial impulse response for the receiving aperture. Both impulse responses are a superposition of spatial impulse responses from the individual elements of a multi-element aperture properly delayed and apodized. Each impulse response is:

$$
h(\vec{r}, t)=\sum_{i=1}^{N_{e}} a_{i}(t) h_{i}\left(\vec{r}_{1}, t-\Delta_{i}(t)\right),
$$

where $a_{i}(t)$ denotes the apodization and $\Delta_{i}(t)$ focusing delay, which both are a function of position in tissue and thereby time. $N_{e}$ is the number of transducer elements.

The received signal from each scatterer must be calculated for each new focusing scheme corresponding to the different lines in an image. The resulting $r f$ signal is then found by summing the responses from the individual scatterers using (1). The number of evaluations of spatial impulse responses for individual transducer elements is:

$$
N_{h}=2 N_{e} N_{s} N_{i}
$$

where $N_{s}$ is the number of point scatterers and $N_{i}$ is the number of imaging directions. It is assumed that the number of elements in both transmitting and receiving aperture are the same, and that the apodization and focusing are included in the calculation. A convolution between $h_{t}\left(\vec{r}_{1}, t\right), h_{r}\left(\vec{r}_{1}, t\right)$ and $v_{p e}(t)$ must be done for each scatterer and each imaging direction. This amounts to

$$
N_{c}=2 N_{s} N_{i}
$$

convolutions for simulating one image.

The same spatial impulse response for the individual elements are, thus, being evaluated $N_{i}$ times for making an image, and an obvious reduction in calculation time can be gained by just evaluating the response once. This can be done by making a synthetic aperture simulation approach. Here the response on each of the receiving elements from excitation of each of the transmitting elements are calculated. The received responses from the individual elements are beamformed afterwards. Hereby the number of evaluations of the spatial impulse responses is

$$
N_{h s}=N_{e} N_{s} .
$$

The number of convolutions is increased to

$$
N_{c s}=N_{s} N_{e}^{2}+N_{s} N_{e}
$$

since all emissions must be convolved with the response from all receiving elements and $v_{p e}(t)$ must be convolved with the responses. This can be reduced to

$$
N_{c s}=N_{s}\left(N_{e}+\sum_{i=1}^{N_{e}} i\right)=0.5 N_{s}\left(N_{e}^{2}+3 N_{e}\right),
$$

if the transmitting and receiving elements are the same, whereby the signal received is the same due to acoustic reciprocity [5], when the transmitting and receiving elements are interchanged. The beamforming is done after the calculation, but this can be done very efficiently as demonstrated in Section 3 . The improvement in calculation of responses is given by

$$
I_{h}=\frac{2 N_{e} N_{s} N_{i}}{N_{e} N_{s}}=2 N_{i}
$$

and for the convolutions

$$
I_{c}=\frac{2 N_{s} N_{i}}{0.5 N_{s}\left(N_{e}^{2}+3 N_{e}\right)}=\frac{4 N_{i}}{\left(N_{e}^{2}+3 N_{e}\right)}
$$

For a 64 element array and an image with 100 directions, the theoretical improvements are $I_{h}=200$ and $I_{c}=0.0933=$ 
1/10.7. The efficiency of the approach is, thus, very dependent on the actual balance between evaluating the spatial impulse responses and performing convolutions. A significant speed-up is attained for few elements and many imaging directions, since few convolutions are performed. The balance is affected by the method for calculating the spatial impulse responses. The simulation program Field II $[6,7]$ offers three different possibilities, which are all based on dividing the transducer into smaller elements. The program uses a farfield rectangle solution [6], a full solution for triangles [8], or a bounding line solution [9]. The first solution is very fast, whereas the last two solutions are highly accurate but significantly slower. The choice of method will, thus, affect the balance.

A second aspect, in the implementation of the approach, is the use of memory. The number of bytes, when using double precision data, is

$$
B_{y}=8 N_{e}^{2} N_{r}
$$

where $N_{r}$ is the number of samples in the response. For at 64 elements array covering a depth of $15 \mathrm{~cm}$, this gives 625 MBytes at a sampling frequency of $100 \mathrm{MHz}$. This is clearly too much for current standard PCs and even for some workstations. The simulation must be made at a high sampling frequency to yield precise results, but the data can, however, be reduced by decimating the signals after simulation of individual responses. A factor of 4 can e.g. be used for a 3 or $5 \mathrm{MHz}$ transducer. The memory requirement is then 156 MBytes, which is more acceptable. It is, however, still large, and much larger than the cache in the computer. It is therefore necessary to reduce the number of cache misses. This is sought achieved in the program by sorting the scatterers according to the distance to the array, which gives results that are placed close in the memory. The memory interface of the computer is, however, very important in obtaining a fast simulation.

A significant reduction can in general be attained with the approach as will be shown later, and the method makes it very easy and fast to try out new focusing schemes once the basic data has been simulated. This would demand a full recalculation in the old approach.

\section{Examples}

All the examples in the following section have been made by a modified version of the Field II simulation system. The parameters used in the simulation are shown in Table 1.

An artificial kidney phantom based on data from the Visible Human Project ${ }^{t}$ has been used as the simulation object. The phantom consists of 200,000 point scatterers within a box of $100 \times 100 \times 35 \mathrm{~mm}$ (lateral, axial, elevation dimension),

\footnotetext{
'Optical, CT and MR images from this project can be found at http://ww.nlm.nih.gov/research/visible/visible_human.html
}

\begin{tabular}{|c|c|l|}
\hline$f_{0}$ & $7 \mathrm{MHz}$ & Transducer center frequency \\
\hline$f_{s}$ & $120 \mathrm{MHz}$ & Sampling frequency \\
\hline$D$ & 3 & Decimation factor for RF data \\
\hline$h_{e}$ & $5 \mathrm{~mm}$ & Height of element \\
\hline pitch & $\lambda / 2$ & Distance between elements \\
\hline$w$ & $0.9 \lambda / 2$ & Width of element \\
\hline$k_{e}$ & $0.1 \lambda / 2$ & Kerf between elements \\
\hline$N_{e}$ & 64 & Number of elements \\
\hline
\end{tabular}

Table 1: Simulation parameters for phased array imaging.

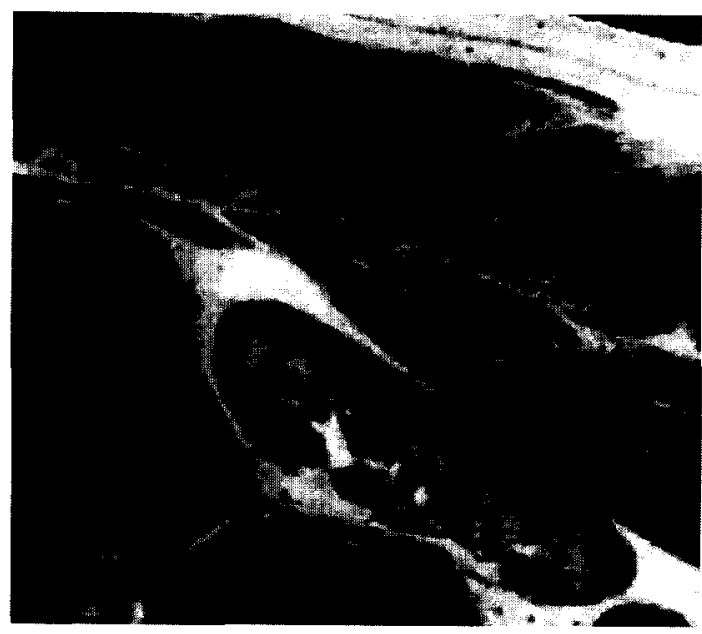

Figure 2: Optical image from the visual human project of a right kidney and a liver lobe.

which gives a realistic size for a full computer simulation of a clinical image. The optical image in Fig. 2 is used for scaling the standard deviation of the Gaussian random amplitudes of the scatterers. The relation between the gray level value in the image and the scatterer amplitude scaling is given by:

$$
a=10 \cdot \exp \left(\operatorname{img}\left(\vec{r}_{k}\right) / 100\right)
$$

where img is the gray-level image data with values from 0 to 127 , and $\vec{r}_{k}$ is the discrete position of the scatterer. This scaling ensures a proper dynamic range in the scatterering from the various structures. The resulting image using a synthetic aperture simulation is shown in Fig. 3

The simulations have been carried out using a standard PC with 512 MBytes RAM and an Athlon $800 \mathrm{MHz}$ CPU running Linux RedHat 6.2. The various simulation times are shown in Table 2. These data also include the beam focusing for the synthetic aperture simulation, which took $9 \mathrm{sec}-$ onds in all cases. It can be seen that the simulation times increase nearly linearly with the number of elements, and linearly with the number of scatterers. The improvement for a phased array image with 128 lines is roughly a factor 5.5 to 6 , which lies between the two boundaries given earlier. The 


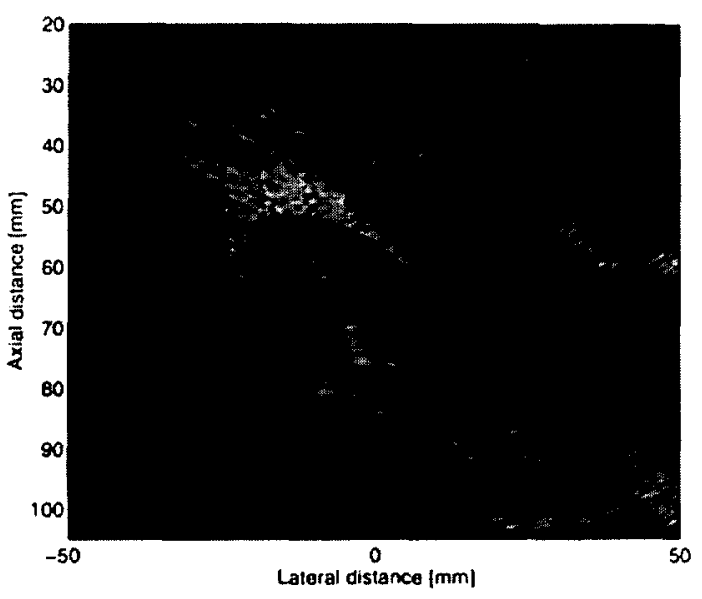

Figure 3: Synthetic ultrasound image of right kidney and liver based on an optical image from the visual human project.

\begin{tabular}{|c|c|c|r|c|}
\hline$N_{s}$ & $N_{e}$ & Method & Time [s] & Improvement \\
\hline 20,000 & 32 & Line & 2944 & - \\
20,000 & 32 & Synthetic & 494 & 5.96 \\
\hline 20,000 & 64 & Line & 6528 & - \\
20,000 & 64 & Synthetic & 1108 & 5.65 \\
\hline 200,000 & 64 & Line & 60288 & - \\
200,000 & 64 & Synthetic & 10972 & 5.49 \\
\hline
\end{tabular}

Table 2: Simulation times for scanning the human-liver phantom.

actual improvement is dependent on the object size, transducer, sampling frequency, $\mathrm{CPU}$, and memory interface, and the numbers will be different for other scan situations.

A three-dimensional scanning has also been implemented. A two-dimensional sparse array ultrasound transducer was used, and a volume consisting of $64 \times 64$ lines with 200,000 scatterers was made. Simulating one line takes 455 seconds, which gives a full simulation time of $1,863,680$ seconds ( 21 days and 13 hours). Using the new approach the whole volume can be simulated in one pass. This takes 6,810 seconds and the beamforming 288 seconds, which in total gives an improvement in simulation time by a factor of 262 . A further benefit is that different focusing strategies also can be tested without a new simulation, and a new volume image can then be made in 288 seconds.

A parallel simulation has also been performed using a Linux cluster consisting of 16 PCs with dual 600 Pentium III processors and 256 MBytes of RAM for every 2 CPUs. The scatterers are then divided into 32 files and the simulation is performed in parallel on all machines. The total simulation time is 935 seconds for the 2D simulation and beamforming takes 9 seconds, when using 200,000 scatterers for the phantom. A full simulation of a clinical image, thus, takes 15 minutes and 44 seconds, which is acceptable for iterative work. This should be compared with 16 hours and 45 minutes on one CPU using the line based simulation. This approach can also be employed for the three-dimensional scanning and can reduce the time for one volume to roughly $212+288=$ 500 seconds. Simulating 15 volumes of data corresponding to one second of volumes for a 3D scanner can then be done in 7,500 seconds or roughly 2 hours.

\section{Acknowledgment}

This work was supported by grant 9700883 and 9700563 from the Danish Science Foundation and by B-K Medical A/S, Gentofte, Denmark.

\section{References}

[1] J. A. Jensen and P. Munk. Computer phantoms for simulating ultrasound B-mode and CFM images. In S. Lees and L. A. Ferrari, editors, Acoustical Imaging, volume 23, pages 75-80, 1997.

[2] A. T. Kerr and J. W. Hunt. A method for computer simulation of ultrasound Doppler color flow images - II. Simulation results. Ultrasound Med. Biol., 18:873-879, $1992 b$.

[3] F. Forsberg, H. Oung, and H. V. Ortega. Doppler simulation for pulsatile flow having nonaxial components. In Proc. IEEE Ultrason. Symp., pages 1713-1716, 1994.

[4] J. A. Jensen. A model for the propagation and scattering of ultrasound in tissue. J. Acoust. Soc. Am., 89:182-191, 1991 a.

[5] L. E. Kinsler, A. R. Frey, A. B. Coppens, and J. V. Sanders. Fundamentals of Acoustics. John Wiley \& Sons, New York, third edition, 1982.

[6] J. A. Jensen and N. B. Svendsen. Calculation of pressure fields from arbitrarily shaped, apodized, and excited ultrasound transducers. IEEE Trans. Ultrason., Ferroelec., Freq. Contr., 39:262-267, 1992.

[7] J. A. Jensen. Field: A program for simulating ultrasound systems. Med. Biol. Eng. Comp., 10th Nordic-Baltic Conference on Biomedical Imaging, Vol. 4, Supplement 1, Part 1:351-353, 1996b.

[8] J. A. Jensen. Ultrasound fields from triangular apertures. J. Acoust. Soc. Am., 100(4):2049-2056, 1996a.

[9] J. A. Jensen. A new calculation procedure for spatial impulse responses in ultrasound. J. Acoust. Soc. Am., pages 3266-3274, 1999. 